\title{
MRI Image Segmentation, Prediction and Diagnostic Accuracy: Deep Learning Framework and Machine Learning Techniques Analysis for Reducing The impact of Cardiac Diseases
}

\author{
R. Kannan R, V. Vasanthi
}

\begin{abstract}
Background: Usage of tele - monitoring system of electronic patient record (EHR) and magnetic reasoning is expected to increase rapidly in near future, yet numerous studies have examined cardiovascular risk prediction and statistic adoptive approach could improve clinical risk prediction.

Objectives: To assess the performance outcomes of various techniques for predicting the risk of cardiovascular diseases and MRI image segmentation method on the basis of systematic review.

Research Design: Retrospective Cardiovascular study. We associate UCI dataset, AHA dataset, real time patient datasets, hospital dataset and sunny broken dataset from 2017 to 2019, and predicted risk using the logistic regression, stochastic gradient boosted, random forest, SVM, ROC Curve, KNN algorithm, MXNET UNET.

Measures: The proposed methods have been developed in four categories to accurately diagnose cardiovascular diseases. We assessed to analyze and compared the accuracy of four different machine learning algorithms with the ROC for assessing and diagnosing cardiovascular disease from UCI cardiac datasets. The research will then focus on to predict heart diseases automatically by segmenting and classifying the patients' heart data in real- time with the help of machine learning algorithms, big data, wireless heart monitor and smart phones. We further improve the prediction accuracy by using logistic regression and ROC Curve to improve the prediction performance. Consequently, $K$ Nearest-Neighbor (KNN) method, $R$ programming language and big data where applied to easily find the nearest hospitals, monitor and provide on-time visualization to the medical professionals. Finally, we propose automatic myocardial segmentation method for cardiac MRI on the basis of Deep Convolutional neural network.
\end{abstract}

Revised Manuscript Received on December 30, 2019.

* Correspondence Author

Dr. V. Vasanthi Assistant Professor in Department of Computer Science, Rathinam College of Arts and Science, Coimbatore, India

Mr. R. Kannan, Ph.D. in Department of Computer Science, Rathinam College of Arts and Science, Coimbatore, India

(C) The Authors. Published by Blue Eyes Intelligence Engineering and Sciences Publication (BEIESP). This is an open access article under the CC BY-NC-ND license (http://creativecommons.org/licenses/by-nc-nd/4.0/)
Results: Logistic Regression methods outperformed the standard accuracy rate even with application of ROC curve (AUC increased from $87 \%$ to $91 \%$ ). Ever better performance was achieved in Models using additional Real time dataset attributes (AUC increased to 93\% and KNN achieved approximately 83\%). Proposed image segmentation method results tended using following techniques, Jaccard $(0.6 \pm 0.1$ mean accuracy Dice's value) outplays the dices co efficient $(0.58 \pm 0.1$ mean accuracy Dice's value) CCN reaches the value of the 0.9 (Table 7) and for the dice's co-efficient respectively that can be compared to manual segmentation. The accuracy tended to decline while PM (Papillary muscles) we got 0.89 for the dice's coefficient and mean squared error 0.01 .

Conclusions: The tele - monitoring system plays the important role for cardiovascular patients and the healthcare industry. Moreover, cardiac image classification demands a high level of expertise and significant time consumption on the part of the operator. Multicenter sufficiently powered and randomized controlled trials are needed to assess the potential benefits and cost-effectiveness of this intervention. Subsequently, our findings of image classification method will facilitate more advanced discovery.

Keywords: Machine learning, Deep learning, logistic regression, KNN algorithm, ROC Curve, Convolutional neural network, Heart disease.

\section{INTRODUCTION}

Currently, the information technology has changed almost every aspect of human life. The use of technology, especially in the health sector is rapidly emphasized and the benefits of this innovative prologue are felt throughout the world. This evolution produces more data on patient which can be worked through technologies and has become machine learning techniques, useful information and knowledge. The data can be used to create expert systems in finding diseases, reducing cost, processing time and improving diagnose. Nevertheless, modern medicine produces large quantities of data every day and has been made to use these available data to solve successfully the challenges ahead [1]. Machine learning plays a tremendous role in healthcare sector and it can process huge data apart from the scope of human capability. It also reliably converts the analysis of those data into medical attention. Recent times, many leading firms have been using machine learning technique to research different types of diseases to predict the early symptoms.

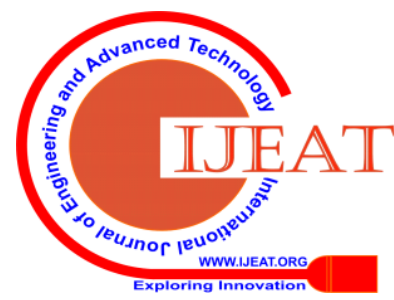


If such information is predetermined, doctors will be able to provide better treatment based on the condition and it shall ultimately lead to better outcomes in favour of patients.

According to statement of World Health Department, Cardiovascular disease is the leading cause of death in the world for the past 10 years and the leading cause of death in high- and low-income countries. Asia and Pacific Economic and Social Commission say that in one fifth of Asian countries, most lives are lost due to non-communicable diseases such as cardiovascular, cancer and diabetes.

A recent study by the Registrar General of India (RGI) and the Medical Research Council of India, reveal that $25 \%$ of deaths in the age group of 25-69 years are due to heart disease. In order to help health physician, researchers use large number of cardiovascular data to extract knowledge they require.

For the past few years, the health communication industry has been playing a key role in efficiently managing and protecting medical data. The data thus collected can help improve the diagnosis and treatment of patients' illnesses. In addition, Health Communication facilitates the systematic management, analysis and use of health-related data for healthcare delivery and service for patients and clients [2].

Researchers have been using various machine learning techniques to diagnose diseases such as diabetes, stroke, cancer and heart diseases. Several Machine learning techniques applied to diagnosis of heart disease have shown different levels of accuracy.

The main contributions of this work are merging with the various machine learning techniques for improved results in diagnose and for prior treatment of heart disease. Since our previous studies present different classification results of presence and absence of cardiac diseases as well as MRI image segmentations [3]. The Proposed system provides optimal results by finding the right combination of different classification and emerging deep learning techniques. With regards to diagnosis approach, this research work proposes three approaches for binary classification of heart diseases based on machine learning techniques [4].

\section{LITERATURE FINDINGS}

we have developed a search strategy for prediction of heart disease and image segmentation form by using the following key words: machine learning, supervised machine learning algorithms, unsupervised machine learning algorithms, gradient boosted, logistic regression, K-Nearest-Neighbor, KNN algorithm, ROC Curve, deep learning, convolutional neural network, UNET, MXNET, MRI Image Segmentation, Big data, disease prediction, heart disease, types of heart diseases, predicting heart diseases, visualizing heart diseases, cardiac diseases, heart diseases prediction experimental and heart image segmentation. We also searched Reference lists of related articles.

\section{LITERATURE REVIEW}

In this section we are going to have comparative study of this research and the works done by other researchers, where machine learning techniques have been used to diagnose heart diseases and convolutional neural network has been used to segment the heart images. The datasets and machine learning algorithm that have been utilized in this research which were applied by various researchers have also found out that various researchers have adopted different techniques such as roc, Naïve bayas, KNN classifiers, SVM, DT,NB convolutional and 5-Fold cross validation, the average dice coefficient layer and eventually have got different results.

In this paper, we are going to elaborately discus about the ROC technique developed by Liangqing Zhang, Cuirong which can be utilized for the purpose of continuous supervision of patients by using a medical monitoring system designed for heart diseases [6]. This method gives outright solutions that consist of data collection, data storage and access, analytical data and feedback. The system also provides prognosis of $\mathrm{HF}$ by the virtue of estimating NT-PROBNP level on the basis of the changes in blood pressure and body weight by using machine learning techniques. The application of machine learning methods yields 29 features of attributes which have been extracted after different sessions of experiments were conducted with the psychological data collection of 7 DAYS and 30 days. A pilot clinical study of 34 samples rends the system more efficient. With the help of this pilot clinical study, NT-Pro BNP Test has been used to help train the prediction model to verify the prediction results for the system. The results obtained by using the systems gave $79.4 \%$ accuracy for predicting HF on day 7 on the basis of daily body weight and blood pressure collected over 30 days.

A number of methods have been proposed in the last decade by Mina Nasr-Esfahani, Majid Mohrekesh to automatically segment the mentioned LV properties by using fully convolutional layer that has been utilized for segmenting left ventricle. Different sessions of experiments were conducted with the York datasets of heart image data collected from 33 Patients. Finally, the Dice score of this method reaches $87.24 \%$ by training effectively the fully convolution layer on the York dataset of heart images.

Wesler et al., According to their systematic review, have used a large number of models that exceeded their requirements. Given the method of comparing individual samples in the past, they provide appropriate guidance on which models are best suited in a given situation. However, the reviews provided earlier were conducted decades ago and excluded models have not been validated internally and externally. Moreover, external articles only describe external values. Therefore, it is imperative to compare how the methods used are appropriate for patient care.

Various prognostic models were developed to assess cardiovascular risk in a systematic study by Temen et al Moreover, they published their reviews of predictive models and their findings in BMJ. They had also examined the development characteristics of the model and if they were subject to external validation. 212 eligible articles were classified as Developmental Articles 125 and External Articles 136.The portrayal of combinations of articles for development and external verification was often cited. 
An approach for tackling data problems was identified in previous researchers and literatures. The proven and proposed method showed good improvements in classification accuracy as compared to the original dataset which contained some missing feature values. The observed improvements for heart disease prediction using a logistic regression algorithm helps predict the signs of a pending heart attack. Receiver Operating Characteristics (ROC) curve in the logistic regression technique improves the prediction performance and the K Nearest-Neighbors (KNN) method to easily find the nearest hospitals. The $\mathrm{R}$ programming language and big data has been effectively utilized for monitoring and providing on-time visualization.

Also on the other hand, previous researchers got different accuracy results for image segmentation method by using fully convolutional network and consequently our system reached good accuracy level.

Table I. UCI Cleveland Dataset

\begin{tabular}{|c|c|}
\hline Variable name & Description \\
\hline Age & Age in years \\
\hline Sex & Sex, 1 for male, 0 for female \\
\hline $\mathrm{CP}$ & $\begin{array}{l}\text { Chest pain type ( } 1=\text { typical angina; } 2= \\
\text { atypical angina; } 3=\text { non-angina pain; } 4= \\
\text { asymptomatic) }\end{array}$ \\
\hline Trestbps & Resting blood pressure \\
\hline Chol & Serum cholesterol in $\mathrm{mg} / \mathrm{d} 1$ \\
\hline Fps & $\begin{array}{l}\text { Fasting blood sugar larger } 120 \mathrm{mg} / \mathrm{dl}(1 \\
\text { true) }\end{array}$ \\
\hline Restecg & $\begin{array}{l}\text { Resting electrocardiographic results }(1= \\
\text { abnormality, } 0=\text { normal) }\end{array}$ \\
\hline Thalach & Maximum heart rate achieved \\
\hline Exang & Exercise-induced angina (1 yes) \\
\hline Old peak & $\begin{array}{l}\text { ST depression induce. Exercise relative to } \\
\text { rest. }\end{array}$ \\
\hline Slope & Slope of peak exercise ST \\
\hline $\mathrm{CA}$ & Number of major vessel \\
\hline Thal & $\begin{array}{l}\text { No explanation provided, but probably } \\
\text { thalassemia }\end{array}$ \\
\hline Num & $\begin{array}{l}\text { Diagnosis of heart disease (angiographic } \\
\text { disease status) } 0(<50 \% \text { diameter } \\
\text { narrowing) } 1(>50 \% \text { diameter narrowing) }\end{array}$ \\
\hline
\end{tabular}

\section{DATA ANALYSIS AND EXTRACTION}

We collected two types of information on presence of cardiovascular diseases and image segmentation method. The data collection techniques acquired for proposed research method are open source datasets and real time data sets. For the purpose of comparison of the data and accurate prediction, following open source data sets have been collected and utilized.

- UCI Cleveland Datasets

- American Heart association

- Sunny Broken Datasets

Three types of datasets have been used as real time sources.
- Heart rhythmic - It consists of the extracted physiological data of patients that has also been used for prediction method (Table 2)

- Patient dataset - it contains the previous health records of the patients which are utilized for even better prediction of heart disease and alert notification to the caretaker when an emergency occurs. (Table 3).

- The Hospital dataset - it includes the data of the hospitals located nearby so that the medical experts can be alerted on time in case of emergency. (Table 4).

As discussed earlier, our primary goal in this study is to predict the performance of heart diseases using Data for this analysis that were taken from the UCI Cleveland datasets (Table 1) repository of healthcare industries which has obtained 303 records with 14 set of variables. An intelligent classification method with those set of variable obtained from UCI Cleveland dataset reveals whether patients have heart disease or not, so that we can predict, alert, and provide visualizations.

Table II. Heart Rhythm Dataset

\begin{tabular}{|l|l|}
\hline Field Name & Description \\
\hline Patient ID & $\begin{array}{l}\text { Patients ID requires to identify and track } \\
\text { the heart diseases by hospitals }\end{array}$ \\
\hline HR & heart rate [bpm] (numeric) \\
\hline Date Time & $\begin{array}{l}\text { date and time (format "YYYY-MM-DD } \\
\text { HH:MI:SS") }\end{array}$ \\
\hline GPS Landmark & $\begin{array}{l}\text { latitude and longitude (GPS X \& Y } \\
\text { coordinates) }\end{array}$ \\
\hline Steps & steps (numeric) \\
\hline GSR & galvanic skin response (numeric) \\
\hline Calories & burned calories (numeric) \\
\hline Temp & skin temperature [ ${ }^{\circ}$ F] (numeric) \\
\hline
\end{tabular}

The prediction model derived by adding new data sets (Heart Rhythmic Dataset, Patient Dataset and Hospital Dataset) is compared with AHA Datasets (Table5) using comparative method for the purpose of improving prediction accuracy and performance of the model.

And even though above - mentioned databases successfully are applied on for early detection of heart diseases, medical professionals are focusing on MRI Scanning methods to improve diagnose efficiency for all Heart diseases. So that we focused on the purpose of MRI Image Segmentation and we utilized Sunny Broken Dataset as they increased the success rate and decreased the decision-making time for the diagnosis of Pending Heart Diseases.

Though there are two other prediction methods, the image segmentation method has adopted to obtain the accurate results and fasten treatment process. For this purpose, sunny brook cardiac data has been utilized to train and evaluate the present network for myocardial segmentation.

Published By:

Blue Eyes Intelligence Engineering

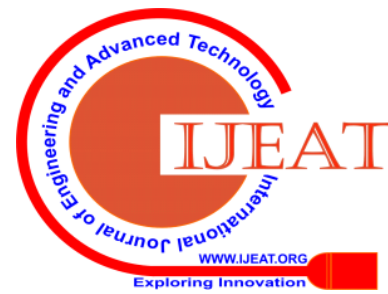


MRI Image Segmentation, Prediction and Diagnostic Accuracy: Deep Learning Framework and Machine Learning Techniques Analysis for Reducing The impact of Cardiac Diseases

The dataset consists of 45 cine MRI images with the expert contours as a part of the clinical routine. Proposed dataset has been obtained from various patients' and pathologists, healthy, hyper trophy, heart failure with infraction and without infraction. For the automatic segmentation of the left ventricle form MRI image, the SCD dataset was used by training MXNET model.

Table III. Patient Dataset

\begin{tabular}{|c|c|}
\hline Field Name & Description \\
\hline Hospital ID & Patient's registered Hospital ID and valid Hospitals listed in Hospitals dataset \\
\hline Patient ID & Patient's ID requires to identify and track the heart diseases by hospitals \\
\hline First Name & Patient First Name \\
\hline Last Name & Patient Last Name \\
\hline Mobile No & Patient's Mobile No to track and extract the data from wireless hrms device \\
\hline Address & Patient's permanent address along with postal code for communication \\
\hline Date of Birth & Valid date $>1900$ and $<=$ Today. \\
\hline Gender & Male/Female/Not known \\
\hline Height & Patient's Height in cm \\
\hline Weight & Patient's Weight in kgs \\
\hline Diagnosis History & The patient's diagnosis existing records \\
\hline Previous Heart failure & $\begin{array}{l}\text { Hypertensive heart disease with heart failure, Ischemic / Dilated cardiomyopathy, } \\
\text { cardiomyopathy, unspecified, heart failure, left ventricular failure, heart failure } \\
\text { unspecified, and applicable }\end{array}$ \\
\hline Smoking status & Never smoked / Ex-smoker / Current smoker \\
\hline Diabetes Status & $\begin{array}{l}\text { Nondiabetic/Diabetics (dietary control)/Diabetic (oral medicine)/Diabetic } \\
\text { (insulin)/Insulin plus oral medication }\end{array}$ \\
\hline Family Member(s) Names & To communicate with the family members when the patients occurs heart \\
\hline $\begin{array}{l}\text { Family Member(s) Mobile } \\
\text { Numbers }\end{array}$ & To alert \& communicate with the family members when the patients occurs heart \\
\hline Wireless HRMS Status & Yes/No \\
\hline Wireless HRMS Number & Wireless heart rating monitoring system unique number \\
\hline Wireless HRMS Name & Wireless heart rating monitoring system Model Name \\
\hline Wireless HRMS Details & Wireless heart rating monitoring system Model technical specification \\
\hline
\end{tabular}


Table IV. Hospital dataset

\begin{tabular}{|l|l|}
\hline Field Name & Description \\
\hline Hospital ID & Registered Unique Hospital ID \\
\hline Hospital Name & Name of the hospital \\
\hline Contact Numbers & Hospital Contact numbers (24/7) \\
\hline Location & Exact Hospital Location \\
\hline GPS landmark & latitude and longitude (GPS X \& Y coordinates) \\
\hline Address & Hospital Address with postal code \\
\hline Working Days & Hospital working days in a week \\
\hline Working Hours & Hospital working hours per day \\
\hline Specialists Name & Names of the heart specialists \\
\hline Specialists Mobile Number & To communicate, identify and track the heart patients diseases \\
\hline Ambulance facility & Yes / No \\
\hline
\end{tabular}

Table V. AHA Predicted Heart Diseases Dataset

\begin{tabular}{|l|l|}
\hline Sinus Rhythm Type & Threshold value of HR, BP and Hotness \\
\hline Normal & $\begin{array}{l}60<=\mathrm{HR}<=100 \text { (Beats } / \mathrm{min}), \mathrm{BP}=100-140 / 60-80 \mathrm{mmHg}, \text { \& Hotness }=36.5- \\
37.5 \circ \mathrm{C}\end{array}$ \\
\hline Bradycardia & $\mathrm{HR}<=60$ (Beats $/ \mathrm{min})$ \\
\hline Tachycardia & $\mathrm{HR}>=100$ (Beats $/ \mathrm{min})$ \\
\hline Hypertension Stage 1) & $\mathrm{BP}=\mathrm{Sys} / \mathrm{Dys}>=140 / 90 \mathrm{mmHg}$ \\
\hline Hypertension (Stage 2) & $\mathrm{BP}=\mathrm{Sys} / \mathrm{Dys}>=150 / 95 \mathrm{mmHg}$ \\
\hline Hypotension & $\mathrm{BP}=\mathrm{Sys} / \mathrm{Dys}<=00 / 60 \mathrm{mmHg}$ \\
\hline Fever & Hotness $>=37.8 \circ \mathrm{C}$ \\
\hline Hypothermia & Hotness $<=35.0 \circ \mathrm{C}$ \\
\hline
\end{tabular}

\section{METHODOLOGY}

To accomplish this goal, we designed three scenarios. 14 sets of variables of the Cleveland dataset risk score (Table 1) age, sex, chest pain type, resting blood pressure, serum cholesterol, fasting blood sugar larger, resting electrocardiographic, maximum heart rate achieved, exercise-induced angina st depression induce exercise relative to res, slope of peak exercise st, number of major vessel No explanation provided, but probably thalassemia and diagnosis of heart disease (angiographic disease status) 0 ( $<50 \%$ diameter narrowing) 1 (>50\% diameter narrowing)) were using multivariable with Four ML Algorithm (Table 6) utilized to find the best cardiac prediction model.
Table VI. Comparison of ACU \& Accuracy Between Models

\begin{tabular}{|l|c|c|}
\hline Algorithms & ACU & Accuracy \\
\hline Logistic Regression & 0.9161585 & 0.8651685 \\
\hline Random Forest & 0.8953252 & 0.8089888 \\
\hline $\begin{array}{l}\text { Stochastic gradient } \\
\text { boosting }\end{array}$ & 0.9070122 & 0.8426966 \\
\hline Support vector machine & 0.882622 & 0.7977528 \\
\hline
\end{tabular}

Table VII. UNET Image Segmentation Accuracy

\begin{tabular}{|l|l|l|}
\hline SF & Architecture & Dice's acc \\
\hline 2 & U-net - Dice's & 0.7491 \\
\hline 2 & U-net - JD & 0.7494 \\
\hline 2 & U-net - BN - JD & 0.8697 \\
\hline 2 & U-net - BN - RI - JD & 0.8899 \\
\hline 1 & U-net - BN - RI - JD & 0.9108 \\
\hline
\end{tabular}


This prediction model was then extended by different tuning parameters and compared to the best model of each machine learning algorithm with ROC curve. The prediction accuracy was tested with a likelihood ratio test for interaction between Roc and Logistic Regression. Moreover, the prediction of the logistic regression method that is compared with the accuracy of ROC yields an accuracy prediction of up to $0.87 \%$ (Figure 1 ).

The improvement of the addition intelligent classification method to diagnose the model was tested with the original variables of real time extracted data - such as (Patient ID ,HR, Date and time, GPS Landmark, Steps, GSR, Calories and Temp ) and AHA datasets (Normal - 60<=HR $<=100$ (Beats /min), BP=100-140/60-80 mmHg, \& Hotness =36.5-37.5 o C , Bradycardia - HR<=60 (Beats $/ \mathrm{min}$ ) , Tachycardia $\mathrm{HR}>=100$ (Beats /min) , Hypertension Stage 1 $\mathrm{BP}=$ Sys/Dys $>=140 / 90 \mathrm{mmHg}$, Hypertension (Stage 2) $\mathrm{BP}=$ Sys/Dys $>=150 / 95 \mathrm{mmHg}$, Hypotension $\mathrm{BP}=$ Sys $/$ Dys $<=00 / 60 \mathrm{mmHg}$, Fever - Hotness $>=37.8$ o C , Hypothermia - Hotness $<=35.0$ o C ) Usage of both datasets was assessed by comparing the predictive method of observed risk to Cleveland datasets based on the logistic regression method to describe the association between response and predictor variables.

The net classification improvement was calculated and quantified with ROC curve to improve accuracy and performance. The method led to a $93 \%$ of prediction accuracy result (Figure 2). Our risk prediction model was based on time-to-event data, which contained not only improved classification and prediction method, but also individual nearest hospital for alerting process in case of emergency occurs by using patient dataset and hospital dataset with KNN Euclidean distance and it has corresponding $83 \%$ of the result of alert processing of SMS calls and email.

Finally, the image segmentation reclassification improvement approach task is demanding and an initialization step is frequently needed to provide more accurate anatomical information to clinical practitioner. The segmentation analyses were performed in sunny broken dataset consists of 45 cine images obtained from various patient and pathologists, healthy, hyper

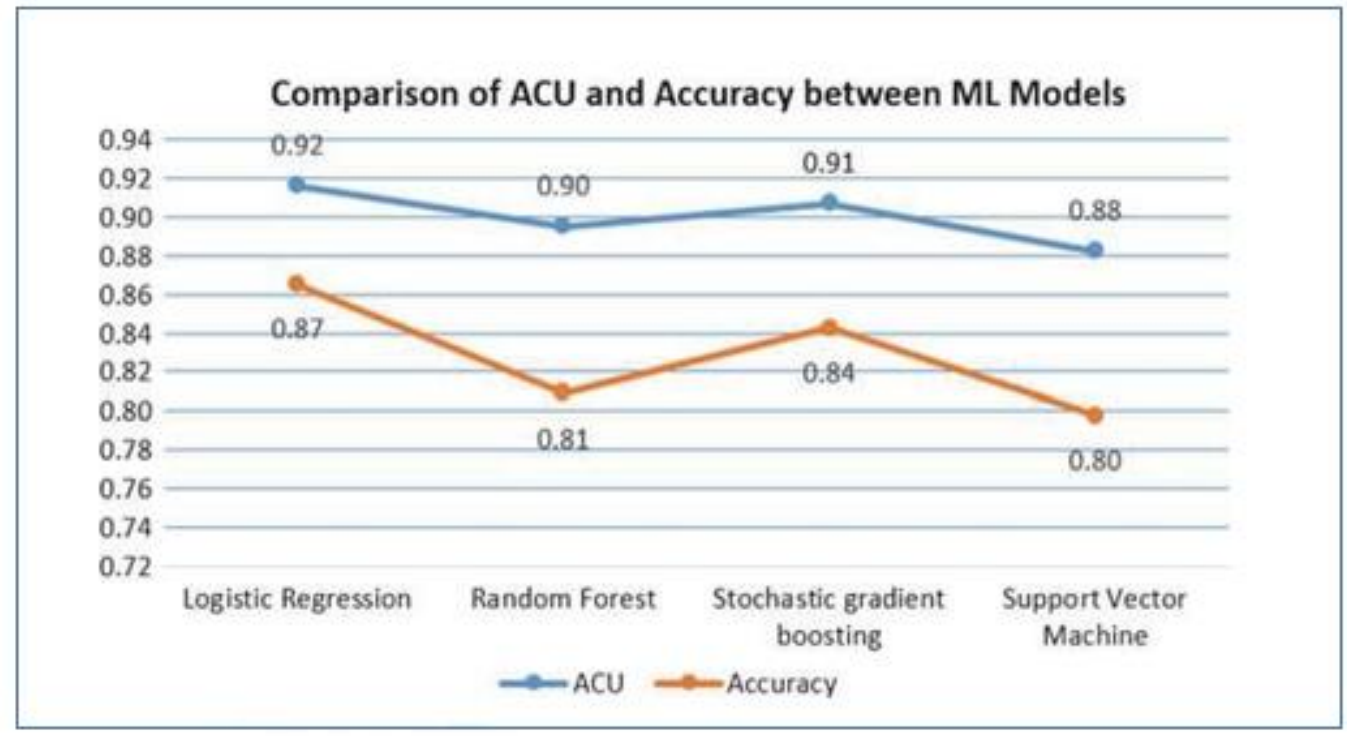

Fig. 1 Visualizing The Results of ACU And Accuracy Models
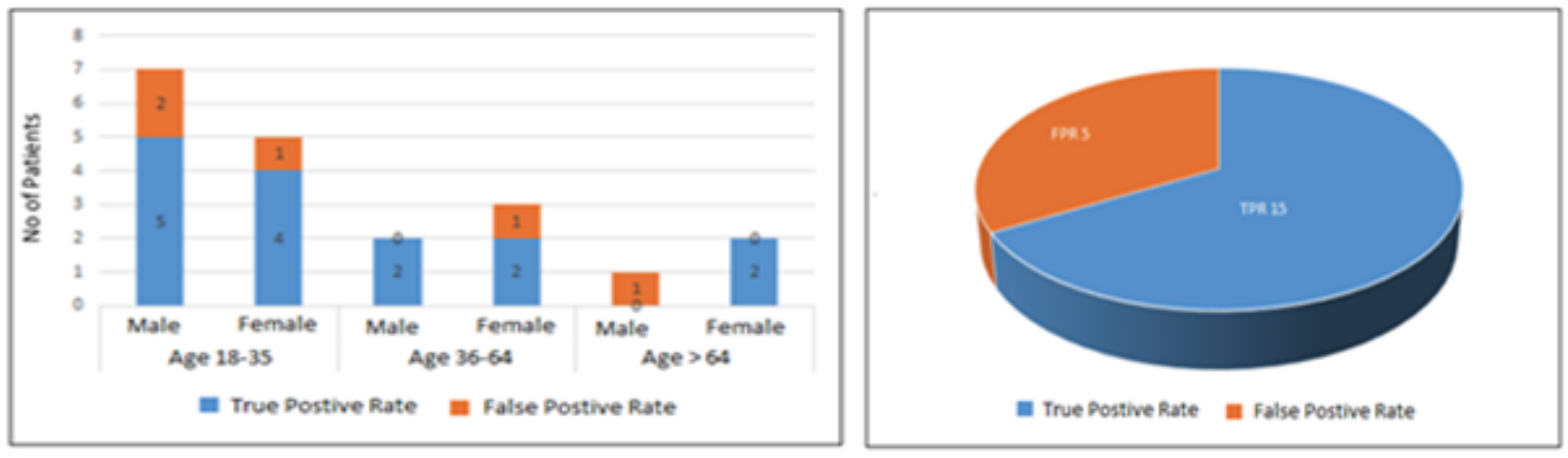

Fig. 2 Heart Diseases Prediction and Performance Measure 


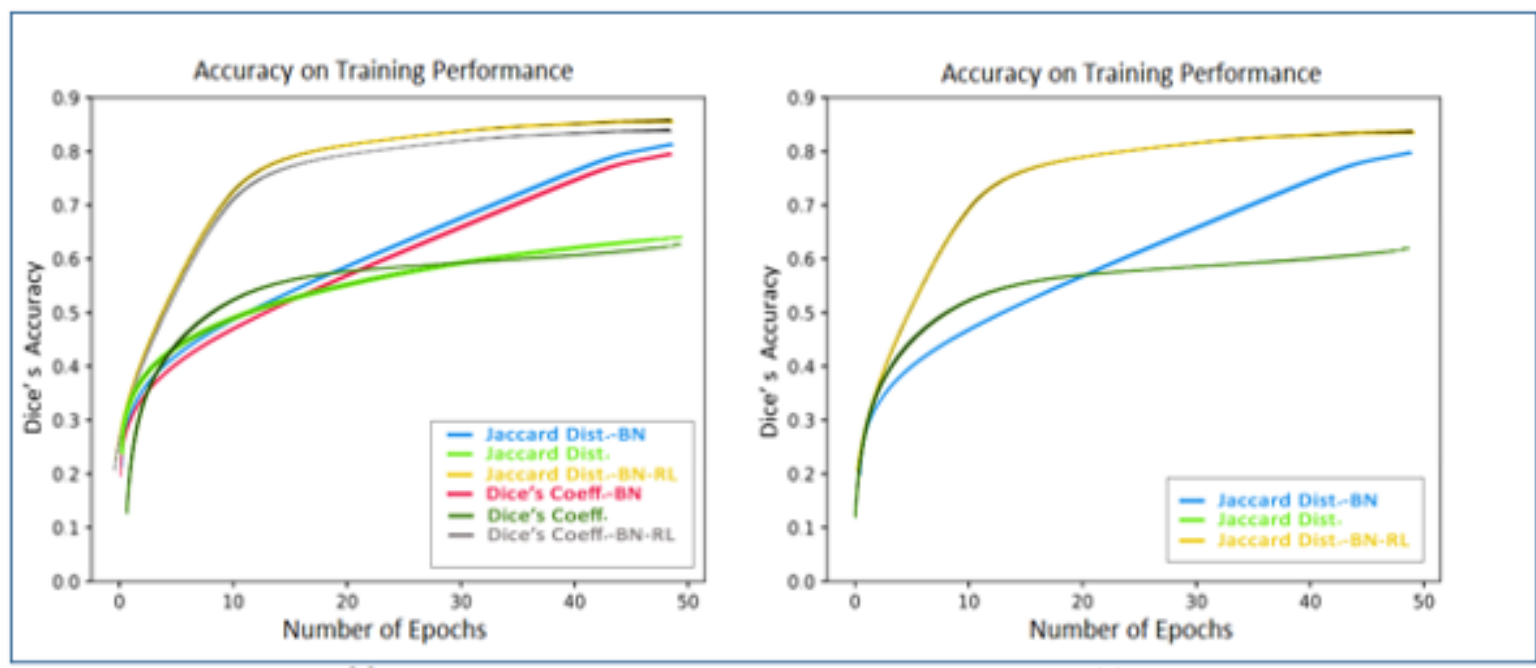

(a)

(b)

Fig 3 Batch Normalization and Residual Learning strategies

trophy, heart failure with infraction and without infraction by training MXNET Model. Specific analysis was performed as images during $10^{\prime}$ to $15^{\prime} \mathrm{sec}$ breath control holds with temporal resolution of 20 cardiac phases over the heart cycle and those images were scanned for the end-diastolic (ED) phase. In order to validate our model, we addressed three sets of experiments were performed for

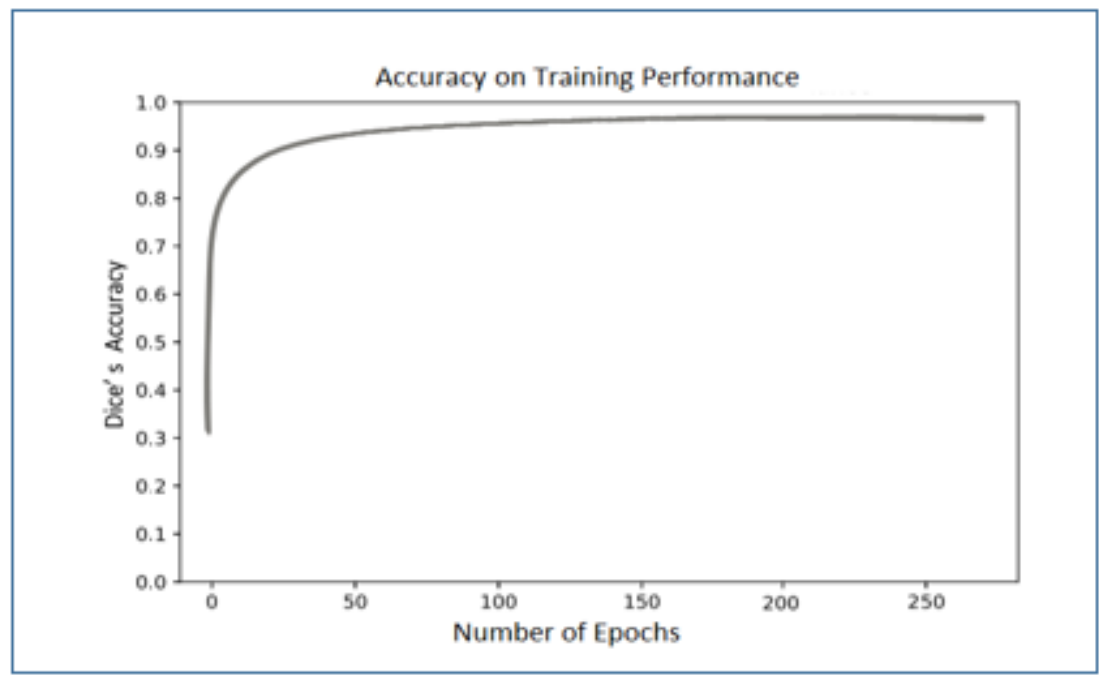

Fig 4 UNET Architecture Accuracy on Training Performance 


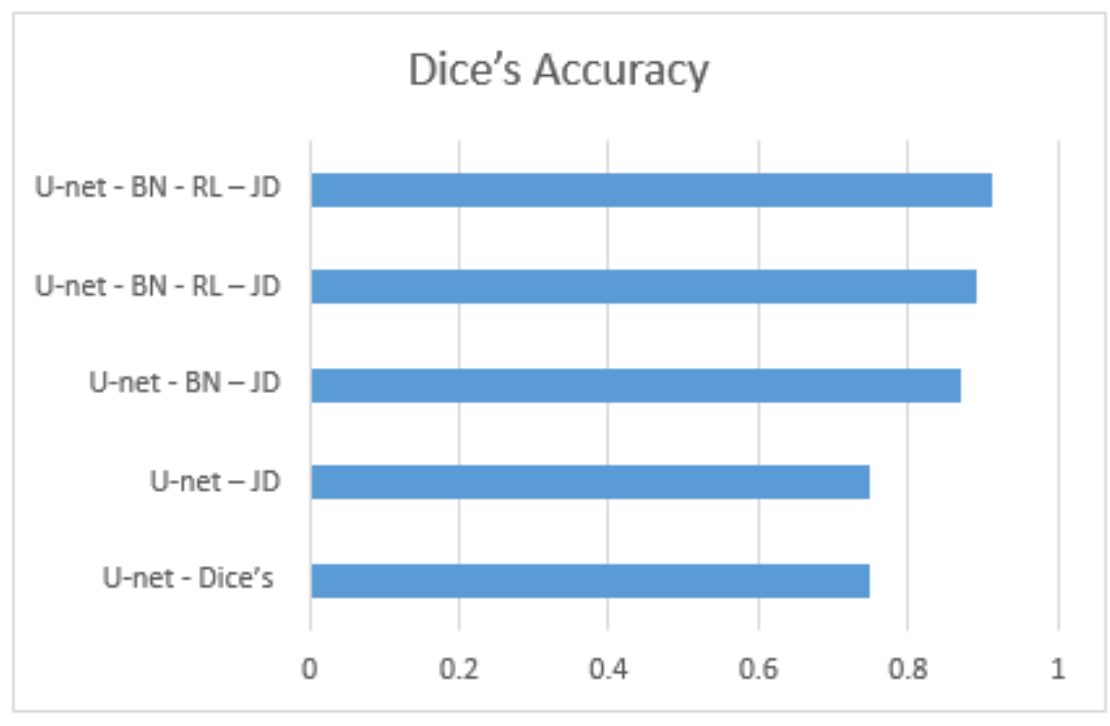

Fig.5 UNET Image Segmentation Accuracy

the purpose of evaluating the methodology on the Sunny Brooks Data. Thus, we developed methods to segment 3 separate outcomes firstly we evaluated the batch normalization, residual learning and the proposed objective function loss (Figure 3), with respect to commonly used dice coefficient on the classic UNET architecture (Figure 4). We have come to know with the help of empirical results (Figure 5), that jaccard (0.6 \pm 0.1 mean accuracy Dice's value) outplays the dices co efficient $(0.58 \pm 0.1$ mean accuracy Dice's value).

\section{DISCUSSION}

\section{A. Limitation}

There are generally several limitations in our studies. The first phase of this study was the usage of open data sources available on the web source, followed by data from a real-time data source and finally an SNCD magnetic rational image, the results of which are not generalizable to other systems. However, we believe that the results of our study accurately reflect the current dataset. The data we have examined may include patients with a variety of diseases such as cardiovascular diseases, respiratory neurological, gastrointestinal, kidney, endocrine, infectious, vascular, traumatic and so may have different effects on MEWS and ML prediction scores. It can also lead to miscalculations. Therefore, future research should be analyzed separately in advance. Another limitation of our study is that although the ML score is shown to have good internal validity according to our data used, external validation of the score for routine clinical usage is required. Finally, the main limitation of this study the lack of a meta-analysis of quantitative data. However, if our objective is to play a key role, this limit does not evaluate the contribution of the review. For future research, further in-depth study of image segmentation should be conducted. In addition, new technologies and application of deep learning in limited periods may be added. In this case, there is no detailed description of how deep neural networks and Machine learning work in the theoretical context. However, given the nature of our goal, review and the understanding of the learners (researchers who do not focus on deep learning in the domain of expertise), such a theoretical approach is not necessarily considered.

\section{B. Findings}

Deep learning in the field of medicine has the benefit of consciousness. Accordingly, the diagnosis is conducted in the medical field using the deep learning method. This includes cardiovascular image segmentation, classification, prognosis, and others, as previously presented. The results of the studies we have reviewed so far show that deep learning methods have higher efficiency as compared to other methods.

Deep learning in the future promises to be useful in various fields of medicine, especially for early detection of diseases. However, at present, it is not clear whether deep learning will change the role of physicians / clinicians in clinical diagnosis. The deep learning system has been good support for therapists. They point to the more widespread usage of deep learning in various fields in different domains such as Netflix, Amazon online services, banking fraudulent, transportation, communication and location service.

According to the literature review cited in this paper, the CNN plays a very important role in accurately specifying the discriminating areas in the cardiac MRI image segmentation. Therefore, the assorted CNN method was used to easily identify pieces with knots. They used discriminatory territorial features to distinguish fragmented parts from complete parts. This method is called a knot activation diagram. Each researcher has used a variety of approaches and data, primarily from CT and CMR images, to illuminate the threshold for cardiac structures. Many authors' approach to heart image segmentation, however, is poorly reporting their methodology and results. It is important to note that only one of the four authors provides an account of their reproductions. Due to infrequent and inaccurate reporting we are unable to provide the abstract and precise results needed for this image extraction system. For this extraction we take 23 heart images and take 7.1 hours 


\section{CONCLUSION}

According to our observation so far, tele monitoring seems to be used in a manner to reduce hospitalization and reading rates for patients with heart failure. This study has shown that tele monitoring alone may not be effective in reducing morbidity and mortality in patients with heart failure. Clearly, the full potential of home tele monitoring is not realized and further clinical trials are needed. However, these data need to be confirmed in larger, long-term studies that are adequately

powered for clinical relevant outcomes. To achieve that we added a system of informing physicians and patient relatives in case of emergency situation.

The tele monitoring system is very useful in reducing the frequency of hospital admissions. It can also be used to prevent the aggravation of heart failure symptoms. Early perception and management of precipitating factors for myocardial infraction such as, infraction or persistent hypertension, new-onset atrial fibrillation, could also considerably reduce admissions. Treatment of beta-blockers, ACE inhibitors and aldosterone antagonists are considered to be very important to improve the health of patients with heart failure caused by left ventricular systolic dysfunction.

In addition, Scientific medical image analysis solves medical problems by analyzing images created in clinical practice. The purpose is to effectively extract information for advanced clinical diagnostics. Recent advances in the field of medicine make medical image analysis one of the bests in research and development areas. An important reason for the advancement of the analysis of these medical images is the optimal usage of machine learning techniques. Deep learning technique has been successfully used as a tool for machine learning where a neural network is capable of automatically learning features. Here, in this paper, we have included details about abnormal diagnosis, early detection of heart disease, heart disease classification, MRI image segmentation and recovery. Moreover, the current state and progress of the Deep Learning System Medical Image Analysis, and the techniques currently used and their challenges are explained.

The heart dieses prediction and the cardiovascular format obtained from medical images such as CT and MRI images are essential step in the progress of patients specific, minimizing the risk of having heart diseases and left ventricle automatic segmentation. This review has been done on the basis of manual and semi automated segmentation techniques that required immense knowledge and considerable amount of

time for the operator. The results finding of this review doesn't defer with the contents of previews authors who have listed the reasons why left ventricle heart image segmentation yet to become a part of routine health care. With reference to these results certain recommendation have been made with regard to left ventricle image segmentation. It's expected that the finding of this research will be instrumental to the growth and motivation of precocious image segmentation techniques.

\section{REFERENCES}

1. Liu, X., Faes L., Kale A et al: A comparison of deep learning performance against healthcare professionals in detecting diseases from medical imaging: a systematic review and meta-analysis, Vol 1(6), 271-297, 2019.

2. Byrne, N., Velasco Forte, M., Tandon, A., Valverde, I., \& Hussain, T. A systematic review of image segmentation methodology, used in the additive manufacture of patient-specific 3D printed models of the cardiovascular system, JRSM Cardiovascular Disease, 2016, https://doi.org/10.1177/2048004016645467

3. Damen Johanna A A G, Hooft Lotty, Schuit Ewoud, Debray Thomas P A, Collins Gary S, Tzoulaki Ioanna et al: Prediction models for cardiovascular disease risk in the general population: systematic review, 2016, BMJ 2016; 353: i2416.

4. Matthew Marsh A, Literature Review of Image Segmentation Techniques and Matting for the Purpose of Implementing "Grab-Cut", Department of Computer Science at Rhodes University, South Africa, Available http://citeseerx.ist.psu.edu/viewdoc/download?doi=10.1 1.103.1334\&rep=rep1\&type $=$ pdf

5. N. J. Dudley, A systematic review of the ultrasound estimation of fetal weight, 80-29, 2015, https://doi.org/10.1002/uog.1751.

6. Mamta Sharma, Farheen Khan, Vishnupriya Ravichandran, "Comparing Data Mining Techniques Used for Heart Disease Prediction, f Engineering and Technology, vol.5, Issue 6, 2017.

7. Vladimir S. Kublanov, Anton Yu. Dolganov, David Belo, and Hugo Gamboa, Comparison of Machine Learning Methods for the Arterial Hypertension Diagnostics, Applied Bionics and Biomechanics, Article ID 5985479, 13, 2017.

8. Sharma, N., \& Aggarwal, L. M. (2010). Automated medical image segmentation techniques. Journal of medical physics, 35(1), 3-14. doi:10.4103/0971-6203.58777.

9. R.Hannah Roseline, R.Jemina Priyadarsini "Retinal Based Disease Prediction using Deep Neural Networks and SVM Classification Techniques", International Journal of Engineering Trends and Technology (IJETT), V49(7),437-444 2017

10. Karim, R., Blake, L., Inoue, J., Tao, Q., Jia, S., Housden, R.J., Bhagirath, P., Duval, J., Varela, M., Behar, J.M., Cadour, L., Geest, R.J., Cochet, H., Drangova, M., Sermesant, M., Razavi, R., Aslanidi, O.V., Rajani, R., \& Rhode, K.S. Algorithms for left atrial wall segmentation and thickness - Evaluation on an open-source CT and MRI image database. Medical Image Analysis, 2018

11. Guo F., Ng M., Wright G. Cardiac MRI Left Ventricle Segmentation and Quantification: A Framework Combining U-Net and Continuous Max-Flow. In: Pop M. et al. (eds) Statistical Atlases and Computational Models of the Heart. Atrial Segmentation and LV Quantification Challenges, Lecture Notes in Computer Science, vol 11395, 2019

12. Mohamed RG, Seada NA, Hamdy S, Mostafa MGM. Automatic liver segmentation from abdominal MRI images using active contours. Int J Comput Appl 2017;176(1):30-37

13. Zhou, Ruan, Canu at al: A review: Deep learning for medical image segmentation using multi-modality fusion, Vol 3, 2019, ttps://doi.org/10.1016/j.array.2019.100004.

\section{AUTHORS PROFILE}

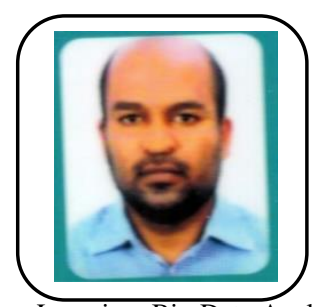

Mr. R. Kannan received Bachelor of Science from Madurai Kamaraj University in 1999, Master of Science from Bharathiyar University in year 2003 and Master of Philosophy from 2004. He is currently pursuing Ph.D. in Department of Computer Science, Rathinam College of Arts and Science, Coimbatore, India since 2017. His main research work focuses on Machine Learning, Deep Learning, Big Data Analytics, Data Mining and Computational Intelligence based education. He has 3 years of teaching experience and 10 years of Industrial Experience 
MRI Image Segmentation, Prediction and Diagnostic Accuracy: Deep Learning Framework and Machine Learning Techniques Analysis for Reducing The impact of Cardiac Diseases

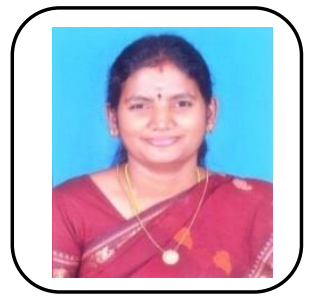

Dr. V. Vasanthi pursed Ph. D from Karpagam University, Coimbatore, Tamil Nadu, India. She is currently working as Assistant Professor in Department of Computer Science, Rathinam College of Arts and Science, Coimbatore, India. She has published more than 20 research papers in reputed international journals including IEEE and Springer and so on. it's also available online. Her main research work focuses on Machine Learning, Network Security, Cloud Security and Privacy, Big Data Analytics, Data Mining, IoT and Computationa Intelligence based education. She has 10 years of teaching experience and 7 years of Research Experience. 